\title{
Non-equilibrium critical behavior of thin Ising films
}

\author{
Maria A. Medvedevaa,*, Pavel V. Prudnikov ${ }^{\mathrm{b}}$, Alexey S. Elin ${ }^{\mathrm{b}}$ \\ ${ }^{a}$ Department of Theoretical Physics and Wave Phenomena, Siberian Federal University, 660041 Krasnoyarsk, Russia \\ ${ }^{b}$ Department of Theoretical Physics, Omsk State University, Omsk 644077, Russia
}

\begin{abstract}
In this paper we study the non-equilibrium properties of Ising ferromagnetic films using Monte Carlo simulations by short-time dynamic method. We have found thickness dependency of critical exponents $z, \theta^{\prime}$ and $\beta / v$. Ageing effects were observed in nonequilibrium critical behavior. Former was carried out both from high-temperature and low-temperature initial states. A characteristic time of relaxation, which diverges at a transition temperature in the thermodynamic limit, is obtained as a function of the system size and waiting time.
\end{abstract}

Keywords: thin film, non-equilibrium critical behavior, aging effects, Monte-Carlo simulation PACS: 68.35.Rh, 68.55.jd, 75.40.Cx, 75.40.Mg

\section{Introduction}

The dynamics of statistical systems close to critical points has been the subject of intensive theoretical and experimental investigations in the last three decades, during which mainly equilibrium properties have been studied in detail. As in the case of static properties, the presence of a nearby critical point greatly facilitates the study of dynamical behaviour. Critical dynamics also provides a simple instance of slow collective evolution. Statistical systems with slow dynamics have recently attracted considerable theoretical and experimental interest, in view of the rich scenario of phenomena they display: dramatic slowing down of relaxation processes, memory effects, etc. After a perturbation a system with slow dynamics does not generically achieve equilibrium even after long times and its dynamics is not invariant either under time translations or under time reversal, as it should be in thermal equilibrium. During this neverending relaxation ageing occurs: two-time quantities such as response and correlation functions depend on the two times $t_{w}$ and $t>t_{w}$ not via $t \rightarrow t_{w}$ only and their decays as functions of $t$ are slower for larger $t_{w}$. At variance with one-time quantities (e.g. the order parameter)converging to asymptotic values in the long-time limittwo-time quantities clearly bear the signature of ageing.

The behaviour of ultrathin magnetic films has become of great technological importance due to the applications in magnetic storage devices [1]. It has been theoretically suggested that the highest areal density advantage for heat assisted magnetic recording (HAMR) can be achieved when the maximum heating temperature is closed to or exceed the Curie temperature [2]. HAMR is the most appropriate technology to achieve magnetic recording densities $1 \mathrm{TBit} / \mathrm{in}^{2}[3,4]$. In this respect,

\footnotetext{
${ }^{*}$ Corresponding author

Email address: mmed@mail.ru (Maria A. Medvedeva)
}

it is important to understand the evolution of magnetization in thin magnetic films, especially at temperatures close to or above the Curie temperature.

In this paper we study the non-equilibrium properties of Ising ferromagnetic films using Monte Carlo (MC) simulations by short-time dynamic method. By exploring the short-time scaling dynamics, we have found thickness dependency of critical exponents $z, \theta^{\prime}$ and $\beta / v$ for ferromagnetic thin film. Ageing effects were observed in non-equilibrium critical behavior. A characteristic time of relaxation, which diverges at a transition temperature in the thermodynamic limit, is obtained as a function of the system size and waiting time.

The paper is organized in the following manner. The Ising model and the Monte Carlo simulation scheme are discussed in the next section (Section 2). The numerical results for universal dimensionality behaviour of thin films with increasing film thickness are given in Section 3. The paper ends with concluding remarks and summary in Section 4.

\section{Model and methods}

In this work we study the ferromagnetic thin film with Ising hamiltonian

$$
H=-J \sum_{<i, j>} S_{i}^{z} S_{j}^{z},
$$

where spins $S_{i}^{z}= \pm 1, J>0$ ferromagnetic exchange constant. The sums are over nearest-neighbor pairs. Periodic and free boundary conditions are used for the in-plane and out-of-plane directions, respectively. The simulations were carried out for systems of size $N_{s}=L \times L \times N$, where $L$ is linear size of layer and $N$ is number of layers. Metropolis algorithm was used for updating spin configurations. 
According to the argument of Janssen et al. [5] obtained with the RG method and $\varepsilon$-expansion, one may anticipate a generalized scaling relation for the $k$-th moment of the magnetization

$$
m^{(k)}\left(t, \tau, L, m_{0}\right)=b^{-k \beta / v} m^{(k)}\left(b^{-z} t, b^{1 / v} \tau, b^{-1} L, b^{x_{0}} m_{0}\right)
$$

And it is realized after a time scale $t_{\text {mic }}$ which is large enough in a microscopic sense but still very small in a macroscopic sense. In (2) $b$ is a spatial rescaling factor, $\beta$ and $v$ are static critical exponents, $z$ is the dynamic exponent, the new independent exponent $x_{0}$ is the scaling dimension of the initial magnetization $m_{0}$ and $\tau=\left(T-T_{c}\right) / T_{c}$ is the reduced temperature.

Since the system is in the early stage of the evolution the correlation length is still small and finite size problems are nearly absent. Therefore, we generally consider $L$ large enough $(L=128)$ and skip this argument.

When system evolving from high-temperature state $\left(m_{0} \ll\right.$ 1) we can choose the scaling factor $b=t^{1 / z}$ and applying the scaling form (2) for $k=1$ to the small quantity $t^{x_{0} / z} m_{0}$, one obtains

$$
\begin{array}{r}
m\left(t, \tau, m_{0}\right) \sim m_{0} t^{\theta^{\prime}} m\left(t^{1 / v z} \tau, t^{x_{0} / z} m_{0}\right)= \\
=m_{0} t^{\theta^{\prime}}\left(1+a t^{1 / v z} \tau\right)+O\left(\tau^{2}, m_{0}^{2}\right)
\end{array}
$$

where $\theta^{\prime}=\left(x_{0}-\beta / v\right) / z$ has been introduced. For $\tau=0$ and small enough $t$ and $m_{0}$, the scaling dependence for magnetization (3) takes the form $m(t) \sim m_{0} t^{\theta^{\prime}}$. For almost all statistical systems studied so far, the exponent $\theta^{\prime}$ is positive. Time scale for this initial increase of magnetization is $t_{0} \sim m_{0}^{-z / x_{0}}$.

For ferromagnetic films the order parameter can be defined as magnetization $m=\left\langle\frac{1}{N_{s}} \sum_{i=1}^{N_{s}} S_{i}\right\rangle$, where angle brackets denote the statistical averaging.

Until now, a completely disordered initial state has been considered as starting point, i.e., a state of very high temperature. The question arises how a completely ordered initial state evolves, when heated up suddenly to the critical temperature. In the scaling form (3), one can skip besides $L$, also the argument

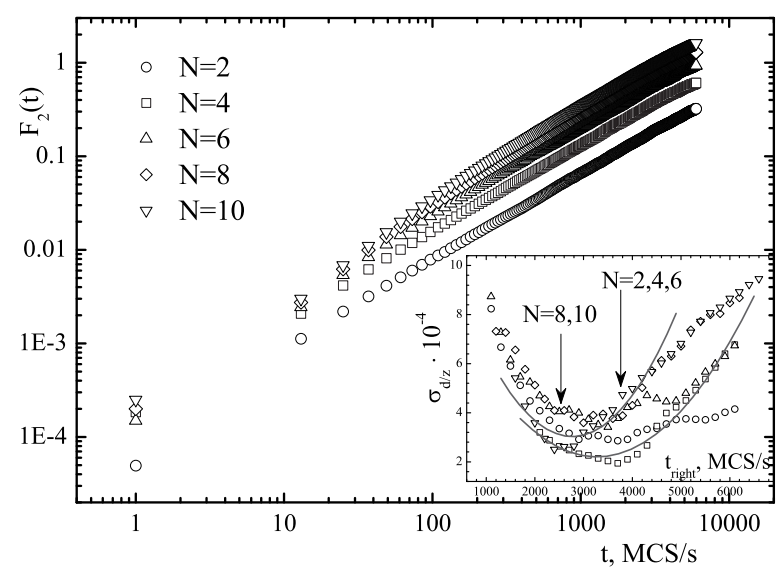

Figure 1: Time dependence of $F_{2}(t)$ at $T=T_{c}, L=128$ $m_{0}=1$

$$
m^{(k)}(t, \tau)=b^{-k \beta / v} m^{(k)}\left(b^{-z} t, b^{1 / v} \tau\right) .
$$

The system is simulated numerically by starting with a completely ordered state, whose evaluation is measured at or near the critical temperature. The quantities measured are $m(t)$ and $m^{(2)}(t)$. With $b=t^{1 / z}$, one avoids the main $t$ dependence in $m^{(k)}(t)$ and for $k=1$ one has

$$
m(t, \tau)=t^{-\beta / v z} m\left(1, t^{1 / v z} \tau\right)=t^{-\beta / v z}\left(1+a t^{1 / v z}+O\left(\tau^{2}\right)\right)
$$

In order to calculate the critical exponent $z$ we have used the ratio [6]

$$
F_{2}(t)=\frac{\left.m^{(2)}(t)\right|_{m_{0}=0}}{\left.m^{2}(t)\right|_{m_{0}=1}} \sim t^{d / z} .
$$

In order to study ageing phenomena we calculated time dependencies of two-time autocorrelation function

$$
\begin{gathered}
C\left(t, t_{w}\right)=\left\langle\frac{1}{N_{s}} \sum_{i} S_{i}(t) S_{i}\left(t_{w}\right)\right\rangle- \\
-\left\langle\frac{1}{N_{s}} \sum_{i} S_{i}(t)\right\rangle\left\langle\frac{1}{N_{s}} \sum_{i} S_{i}\left(t_{w}\right)\right\rangle,
\end{gathered}
$$

where $t$ is the time from of the sample preparation; $t_{w}$ ("waiting time") is time which characterizes the time elapsed since the preparation of the sample prior to measurement of its quantities.

In the pure (undiluted) $3 \mathrm{~d}$ Ising model the critical slowing down, i.e. an increase of the autocorrelation time $\tau_{\text {cor }}$ as the critical point $T_{c}$ is approached, is governed by the universal dynamical critical exponent $z$

$$
\tau_{\text {cor }} \sim\left|T-T_{c}\right|^{-v z}
$$

with the correlation length critical exponent $v$.

Below we briefly review MC studies performed so far to analyze how the relation (8) holds for the Ising model and, in particular, to answer the question how the dynamical critical exponent $z$ is influenced by $t_{w}$ and film thin $N$.

One may consider that the critical temperature as well as the critical exponents associated with the transition can be easily obtained by the relaxation time near the critical temperature. When the temperature approaches the critical point $T_{c}$, the relaxation time $\tau$ diverges as (8). Thus, we can estimate dynamic critical exponents $z$ using the finite-size scaling for the relaxation times $\tau\left(L, T_{c}\right) \sim L^{z}$. There are, however, several difficulties in obtaining the relaxation time by MC simulations. One difficulty is due to extremely slow dynamics of our systems as mentioned above. Another difficulty is that the method of obtaining the relaxation time requires accurate estimation of a long-time tail of the autocorrelation function.

In order to avoid the above-mentioned difficulties, Bhatt and Young [7] have introduced a dimensionless dynamic correlation function

$$
R\left(t, t_{w}\right)=\frac{C\left(t, t_{w}\right)}{\sqrt{\left[\left\langle\left(\frac{1}{N_{s}} \sum_{i} S_{i}(t) S_{i}\left(t_{w}\right)\right)^{2}\right\rangle\right]}} \sim e^{-\delta t / \tau_{\mathrm{cor}}}
$$



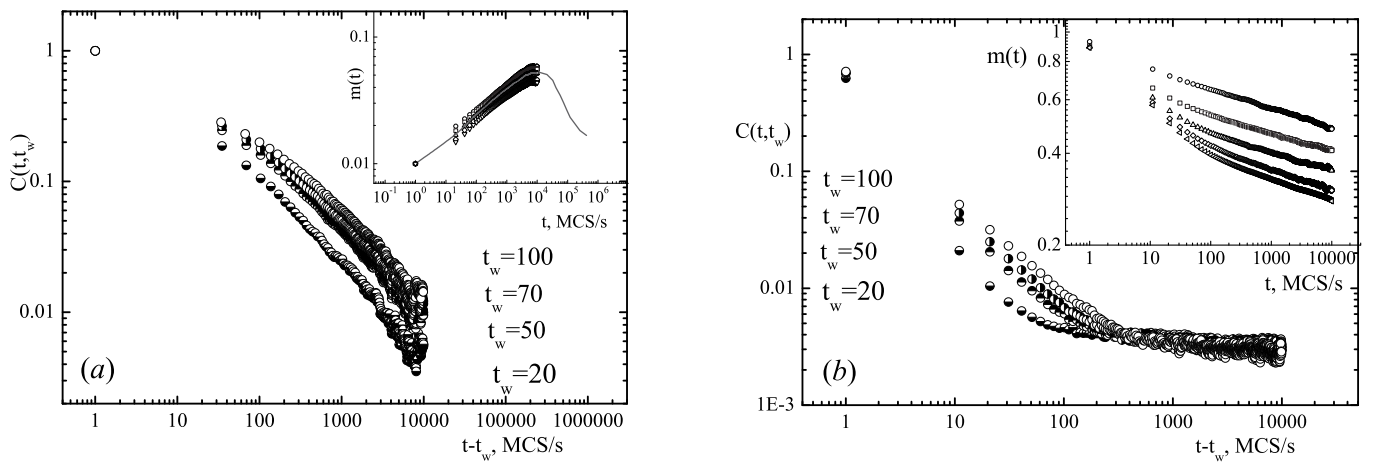

Figure 2: Time dependencies of autocorrelation function $C\left(t ; t_{w}\right)$ for $N=2$ for different $t_{w}$ and initial state $m_{0} \ll 1(a), m_{0}=1(b)$. Time dependencies of magnetization for $N=2(\circ), 4(\square), 6(\triangle), 8(\diamond), 10(\nabla)$.

Equation (9) is the ratio of an odd moment of the autocorrelation function to an even one. Consequently, the ratio functions for a given $t_{w}$ decay from 1 at $t=0$ to zero as $t$ goes to infinity.

\section{Results of Monte Carlo modeling}

\subsection{Dimensionality effects in critical behaviour of ultrathin films}

For calculating the critical exponents $z$ and $\theta^{\prime}$ of ferromagnetic films we considered systems with linear size $L=128$ and layers number $N=2 ; 4 ; 6 ; 8 ; 10$. We have studied evolution of thin film both from high-temperature $\left(m_{0} \ll 1\right)$ (Fig. 2a) and from low-temperature $\left(m_{0}=1\right)$ (Fig. 2b) initial states. The solid line corresponds to behaviour of magnetization predicted by Janssen et al. [5]. Starting from initial configurations, the system was updated with Metropolis algorithm at the critical temperatures $T_{c}=3.2076(4)$ for $N=2, T_{c}=3.8701$ (3) for $N=4, T_{c}=4.1179(3)$ for $N=6, T_{c}=4.2409$ (2) for $N=8$ and $T_{c}=4.3117(3)$ for $N=10$ [8]. Simulations have been performed up to $t=10000 \mathrm{MCS} / \mathrm{s}$. One would like to mention that measurements starting from a low-temperature initial state $\left(m_{0}=1\right)$ are more favorable, since they are much less affected by fluctuations, because the quantities measured are rather big in contrast to those from a random start with zero or small initial magnetization $\left(m_{0} \ll 1\right)$.

We have measured the time evolution of the magnetization $m(t)$ and the second moment $m^{(2)}(t)$ from high-temperature and low-temperature initial states. It allowed to calculate the timedependent cumulant $F_{2}(t)$ (Fig. 1). In Fig. 2 the magnetization is plotted for a high-temperature initial state (Fig. 2a) and a lowtemperature initial state (Fig. 2b) for different layer number $N=2,4,6,8,10$.

The increasing of the magnetization $m(t)$ from a hightemperature initial magnetization $m_{0} \ll 1$ at short-time regime $t<t_{c r} \simeq m_{0}^{-1 /\left(\theta^{\prime}+\beta / z v\right)}$ have the form $m(t) \sim t^{\theta^{\prime}}$.

The initial rise of magnetization is changed to the wellknown decay $m(t) \sim t^{-\beta / v z}$ for $t \gg t_{c r}$. From slope of magnetization at high-temperature initial state on $t \in[100 ; 3500]$ we can estimate the value of critical non-equilibrium exponent $\theta^{\prime}$. From the slope of time dependence of the cumulant $F_{2}(t)$ we can estimate the value of critical exponent $z$. The calculated values of $z, \theta^{\prime}$ and $\beta / v$ are presented in Table 1 . The dependence of the mean square error $\sigma_{d / z}$ as a function of the right border of the time interval $\delta t \in\left[100 ; t_{\text {right }}\right]$ is presented in Fig. 1 for different thin film. The time interval $\left[100 ; t_{\text {right }}\right]$ for $t_{\text {right }}=3800$ gives the minimum of errors for exponent $d / z$ for film thickness $N=2,4,6$. The time interval $\left[100 ; t_{\text {right }}\right]$ for $t_{\text {right }}=2500$ gives the minimum of errors for exponent $d / z$ for film thickness $N=8,10$. Comparing the calculated values with the results for 2D Ising model in various works, we see the very good agreement among them, ie thin ferromagnetic films belong to universality class non-equilibrium behavior of 2D Ising model.

\subsection{Ageing effects}

In order to research ageing phenomena we have calculated two-time autocorrelation function. Time dependencies of autocorrelation function $C\left(t, t_{w}\right)$ are presented in Fig. 2. Autocorrelation function $C\left(t, t_{w}\right)$ for $t_{w}=20,50,70,100 \mathrm{MCS} / \mathrm{s}$ and for $N=2$ are presented in Fig. 2(a) for evolving from hightemperature initial state and 2(b) for low-temperature initial state. The resulting curves presented in Fig. 2 have been obtained by averaging over 30000 samples.

Table 1: Values of the critical exponents $z, \theta^{\prime}$ and $\beta / v$

\begin{tabular}{|c|c|c|c|}
\hline System & $z$ & $\theta^{\prime}$ & $\beta / v$ \\
\hline Present report & $\mathrm{N}=2: 2.095(12)$ & $0.200(2)$ & $0.129(2)$ \\
\hline $\mathrm{MC}$ & $\mathrm{N}=4: 2.106(15)$ & $0.197(3)$ & $0.127(2)$ \\
\hline & $\mathrm{N}=6: 2.153(14)$ & $0.173(2)$ & $0.126(3)$ \\
\hline & $\mathrm{N}=8: 2.154(13)$ & $0.185(3)$ & $0.154(3)$ \\
\hline & $\mathrm{N}=10: 2.158(20)$ & $0.163(3)$ & $0.155(5)$ \\
\hline & \multicolumn{3}{|c|}{ 2D Ising } \\
\hline MC, 1997 [9] & & $0.191(1)$ & \\
\hline RG, $2006[10]$ & $2.0842(39)$ & & \\
\hline MC, 1997 [11] & $2.166(7)$ & & \\
\hline MC, 2000 [12] & $2.1667(5)$ & & \\
\hline MC, 1984 [13] & $2.14(2)$ & & \\
\hline MC, 1983 [14] & $2.12(6)$ & & \\
\hline
\end{tabular}


Autocorrelation function have scaling form

$$
C\left(t, t_{w}\right) \sim t_{w}^{-2 \beta / v z} F_{C}\left(t / t_{w}\right) .
$$

Data presented in Fig. 2 clear demonstrates the presence of the two characteristics regimes: quasi-equilibrium regime at times $\left(t-t_{w}\right) \ll t_{w}$ and non-equilibrium regime at times $\left(t-t_{w}\right) \gg$ $t_{w}$. At times $\left(t-t_{w}\right) \sim t_{w}$ there is a crossover regime with the dependence of correlation characteristics of the waiting time.

At quasi-equilibrium regime autocorrelation function have a scaling form $C(t) \sim t^{-c_{a}}$, where $c_{a}=\gamma / z v$. As we can see on Fig. 2 the increasing of system age $t_{w}$ lead to decreasing of value $c_{a}$ and consequently lead to amplification of critical slowdown and ageing effects.

In order to estimate time correlation of our systems we calculate dimensionless dynamic correlation function $R\left(t, t_{w}\right)(9)$ for different film thickness $N=2,4,6,8,10$ and for different waiting time $t_{w}=20,50,70,100$. Time dependencies of dimensionless dynamic correlation function $R\left(t, t_{w}\right)$ are presented in Fig. 3. At times large enough $R\left(t, t_{w}\right)$ decays exponentially (9). Values of autocorrelation time presented in the table 2 for different thickness and waiting time.

We expect that the ratio functions pick up only slowest relaxation modes from the whole modes of $C\left(t, t_{w}\right)$. A typical example of the ratio function, as well as $C\left(t, t_{w}\right)$, is presented for Ising ferromagnetic film in Fig. 3. As expected, the ratio functions are unity in the relatively short-time regime, implying that the denominator and the numerator coincide with each other. For the longer-time regime, as shown in Fig. 3, the ratio functions of spin sector coincide with the tail of the corresponding autocorrelation function multiplied by a certain constant, which represents a statistical weight of the slowest modes in $C\left(t, t_{w}\right)$.

\section{Conclusions}

The time dependencies of magnetization and cumulant $F_{2}(t)$ have been studied for Ising thin film with using short-time dynamics method for different sizes of film.

The dimensional crossover of magnetic properties from twodimensional to three-dimensional character in magnetic multilayers has currently attracted much interest as a result of both technological and fundamental importance. Of particular interest is the critical behavior of magnetic thin films for which the dimensionality $d$ is not well established. This crossover had been observer in behaviour of critical exponent $\beta$. It was obtained in paper devoted to the experimental study of the critical behavior of thin films [15] and MC simulation of anisotropic Heisenberg model $[16,17]$. Dependence of critical exponents $z, \theta^{\prime}, \beta / v$ from film thickness was obtain. For film with $N=2-6$

\begin{tabular}{|l|l|l|l|l|}
\multicolumn{5}{|c}{ Table 2: Values of the autocorrelation time } \\
\hline$N$ & $t_{w}=20$ & $t_{w}=50$ & $t_{w}=70$ & $t_{w}=100$ \\
\hline 2 & $21315(91)$ & $35173(120)$ & $38950(110)$ & $47317(117)$ \\
\hline 4 & $25999(76)$ & $34677(93)$ & $39295(88)$ & $46612(87)$ \\
\hline 6 & $24269(82)$ & $37277(130)$ & $42624(129)$ & $46538(139)$ \\
\hline 8 & $11632(46)$ & $16866(56)$ & $22315(64)$ & $26189(66)$ \\
\hline
\end{tabular}

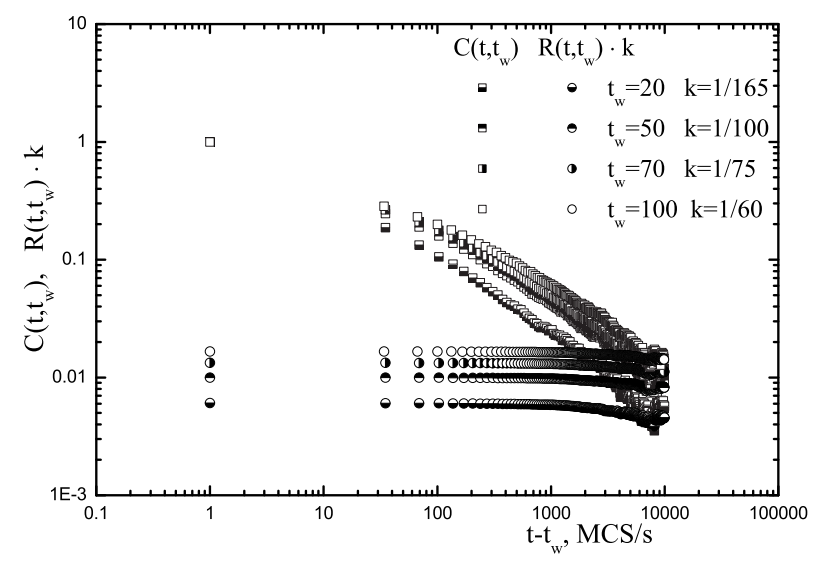

Figure 3: The log-log plot for the ratio function $R\left(t, t_{w}\right)$ and autocorrelation function $C\left(t, t_{w}\right)$ for $L=128, N=2, t_{w}=20,50,70,100, m_{0} \ll 1$.

values are the same within the error. These systems belong to the same universality class. Another feature of non-equilibrium behaviour in system with slow dynamics if aging effect. This effect has been observed in this system. Value of autocorrelation time demonstrate existence of aging effect in our system. The increasing of system age $t_{w}$ lead to increasing of value $\tau_{c o r}$.

\section{Acknowledgments}

The work was performed within the grant No. MD6024.2016.2 of Russian Federation President and within the project of Russian Fund of Basic Research No. 16-32-00581 mol_a. The simulations were supported by the Supercomputing Center of Lomonosov Moscow State University, Moscow Joint Supercomputer Center and St. Petersburg Supercomputer Center of the Russian Academy of Sciences.

\section{References}

[1] C. Chappert, A. Fert and F. Nguyen van Dau, Nature Mater. 6 (2007) 813.

[2] A. Lyberatos and K.Y. Guslienko, J. Appl. Phys. 94 (2003) 1119.

[3] R. E Rottmayer, S. Batra, D. Buechel, et al., IEEE Trans. Magn. 42 (2006) 2417.

[4] M. A. Seigler, W. A. Challener, et al., IEEE Trans. Magn. 44 (2008) 119.

[5] H.K. Janssen, B. Schaub and B. Schmittmann, Z. Phys. B 73 (1989) 539.

[6] R. da Silva, N. A. Alves and J. R. Drugowich de Felécio, Phys. Lett. A 298 (2002) 325.

[7] R.N. Bhatt, A.P. Young, Europhys Lett. 20 (1992) 59.

[8] Y. Laosiritaworn, J. Poulter and J.B. Staunton, Phys. Rev. B 70 (2004) 104413-1.

[9] K. Okano, L. Schülke, K. Yamagishi and B. Zheng, Nucl. Phys. B 485 (1997) 727.

[10] A. S. Krinitsyn, V. V. Prudnikov and P. V. Prudnikov, Theor. Math. Phys. 147 (2006) 561.

[11] F.-G. Wang and C.-K. Hu, Phys. Rev. E 56 (1997) 2310.

[12] M. P. Nightingale and H. W. Blöte, Phys. Rev. B 62 (2000) 1089.

[13] C. Kalle, J. Phys. A 17 (1984) 801.

[14] N. Jan, L. Leo Moseley and D. Stauffer, J. Stat. Phys. 33 (1983) 1.

[15] Y. Li and K. Baberschke, Phys. Rev. Lett. 68 (1992) 1208.

[16] P.V. Prudnikov, V.V. Prundikov, M.A. Medvedeva, JETP Lett. 100 (2014) 501. 
[17] P.V. Prudnikov, M.A. Menshikova et al., J. Magn. Magn. Mater. 387 (2015) 77. 